Document downloaded from:

http://hdl.handle.net/10251/145200

This paper must be cited as:

Gregori Gregori, V.; Miñana, J.; Miravet-Fortuño, D. (15-0). Contractive sequences in fuzzy metric spaces. Fuzzy Sets and Systems. 379:125-133.

https://doi.org/10.1016/j.fss.2019.01.003

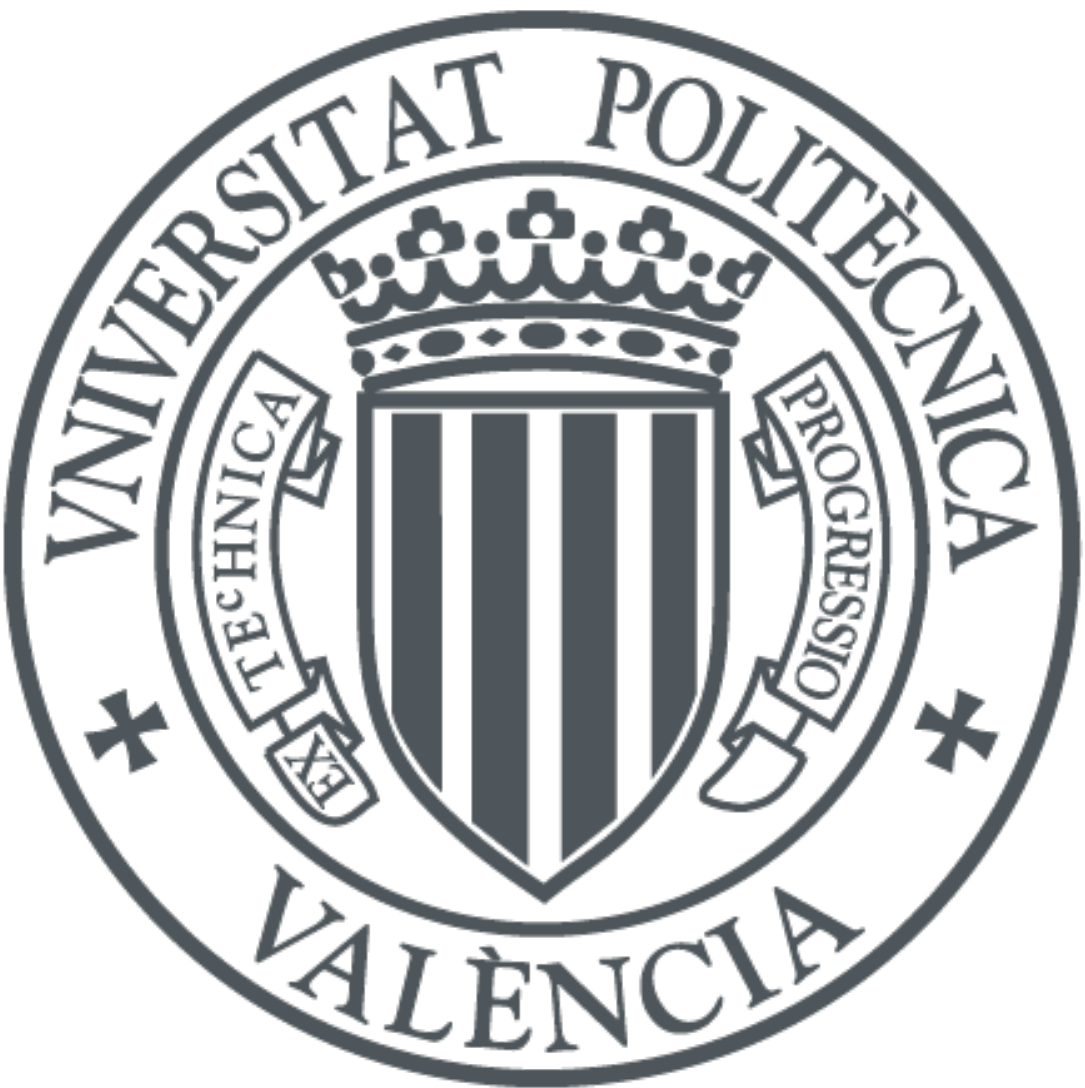

The final publication is available at

https://doi.org/10.1016/j.fss.2019.01.003

Copyright Elsevier

Additional Information 


\title{
Contractive sequences in fuzzy metric spaces
}

\author{
Valentín Gregori $^{1}$, Juan-José Miñana ${ }^{2}$, David Miravet ${ }^{1}$ \\ ${ }^{1}$ Universitat Politècnica de València, Instituto de Investigación para la Gestión Integrada \\ de Zonas Costeras, Campus de Gandia, C. Paranimf 1, 46730 (Spain). \\ vgregori@mat.upv.es,damifor@alumni.uv.es \\ ${ }^{2}$ Departament de Ciències Matemàtiques i Informàtica \\ Universitat de les Illes Balears \\ Carretera de Valldemossa km. 7.5, 07122 Palma (SPAIN) \\ jj.minana@uib.es
}

\begin{abstract}
In this paper we present an example of a fuzzy $\psi$-contractive sequence in the sense of D. Mihet, which is not Cauchy in a fuzzy metric space in the sense of George and Veeramani. To overcome this drawback we introduce and study a concept of strictly fuzzy contractive sequence. Then, we also make an appropriate correction to Lemma 3.2 of [V. Gregori and J. Miñana, On fuzzy $\psi$-contractive sequences and fixed point theorems, Fuzzy Sets and Systems 300 (2016), 93-101].
\end{abstract}

Keywords: Fuzzy metric space, Fuzzy contractive mapping, Fixed point 2010 MSC: 54A40, 54D35, 54E50

\section{Introduction}

In this paper we deal with the concept of fuzzy metric due to George and Veeramani [1] which is a modification of the one given by Kramosil and Michalek [9, 3]. If $M$ is a fuzzy metric on $X$ then $M$ generates a topology $\tau_{M}$ on $X$ in a similar way to classical metrics. This topology is metrizable [2, 7] and consequently, topics related to metrics have been systematically extended and studied in this fuzzy setting. In particular, (fuzzy) fixed point theory is a field of high activity.

Recall that in classical fixed point theory, a contractive sequence of iterates $\left\{f^{(n)}\left(x_{0}\right)\right\}$ of a self-contractive mapping $f$ on a complete metric space $X$ is constructed, for all $x_{0} \in X$. This sequence converges in $X$ since contractive sequences are Cauchy. But, what about this statement in fuzzy setting? 
We notice that in [8] the authors introduced a concept of fuzzy contractive sequence and they posed the following question: Is every fuzzy contractive sequence a Cauchy sequence? So far, there is no answer to this question (D. Mihet [11] gave a negative answer, but for fuzzy metrics in the sense of Kramosil and Michalek). The purpose of this article is to make a new contribution to this field and, at the same time, to correct an error appeared in [5]. For it, we will introduce and study a concept of strictly fuzzy contractive sequence.

Regarding the last paragraph, on the one hand, we notice that there are several concepts of Cauchy sequence in the literature [6]. Here we focus our attention in the two concepts used in fuzzy fixed point theory. The first one was given by M. Grabiec in 3 and it will be denoted by $G$-Cauchy (see Definition 2.2). The second one will be called, simply, Cauchy (see Definition 2.3) and it is due to George and Veeramani [1] (although it comes from PM-spaces [13]). It is well known that Cauchy implies G-Cauchy. On the other hand, with respect to (fuzzy) contractive mappings, we deal with four related concepts, named within brackets, due (chronologically) to Gregori and Sapena (GS) [8], Mihet ( $\psi$-contractive) [11, Romaguera and Tirado (RT) [12], and Wardowski $(\mathcal{H})$ [15] (see Definition 2.4). The relationship among these concepts is shown in the following chain of (strict) implications:

RT-contractive $\Longrightarrow$ GS-contractive $\Longrightarrow \mathcal{H}$-contractive $\Longrightarrow \psi$-contractive

According to these concepts, and in a similar way to classical metrics, we obtain their corresponding concepts of (fuzzy) contractive sequence (see Definition 2.5), which preserve the aforementioned chain of implications. In this paper we study which of these contractive conditions implies Cauchyness.

We observe in Proposition 3.11 that every $\psi$-contractive sequence is $G$ Cauchy. Nevertheless, our main result is Example 3.12 in which we construct a $\psi$-contractive sequence in a stationary fuzzy metric, which is not Cauchy. This example points out that the concept of $\psi$-contractivity needs to be strengthened, to some strictly fuzzy contractivity, to get Cauchyness. But in what form should it be done? Our decision is based on (the proof of) Lemma 3.2 of [5], which asserts: "A $\psi$-contractive sequence $\left\{x_{n}\right\}$ satisfying $\bigwedge_{t>0} M\left(x_{1}, x_{2}, t\right)>0$, is a Cauchy sequence". It is clear, at the light of Example 3.12, that this lemma is false. The error in the mentioned proof is due to the fact that the authors have improperly used the property: $M\left(x_{m+1}, x_{n+1}, t\right) \geq \psi\left(M\left(x_{m}, x_{n}, t\right)\right)$, for all $m, n \in \mathbb{N}$. Therefore we 
just define a strictly fuzzy $\psi$-contractive sequence (see Definition 3.2) as a $\psi$-contractive sequence that satisfies that property. In this manner, the mentioned Lemma 3.2 is valid for strictly fuzzy $\psi$-contractive sequences satisfying $\bigwedge_{t>0} M\left(x_{1}, x_{2}, t\right)>0$.

In a similar way, the other three concepts of strictly fuzzy contractive sequence are defined, and among these four concepts, again the above chain of implications is satisfied. The given concept of strictly fuzzy contractive sequence can be considered an appropriate concept, not only because it makes true Lemma 3.2 aforementioned, but also because for $x_{0} \in X$ the sequence of iterates $\left\{f^{(n)}\left(x_{0}\right)\right\}$ of a contractive mapping $f$, for each one of the mentioned contractive conditions, is strictly fuzzy contractive (see Proposition 3.5). The reader can find another favorable argument to this new concept in Proposition 3.7. Moreover, in (b) of Example 3.9 we give a convergent $R T$-contractive sequence, which is not strictly $R T$-contractive.

After properly correcting Corollary 3.8 and Lemma 3.12 of [5] we show two large classes of fuzzy metric spaces where the condition of strictly fuzzy contractivity for a sequence implies Cauchy.

The structure of the paper is as follows. Section 2 is dedicated to preliminaries. Section 3 contains the concept of strictly fuzzy $\psi$-contractive sequence and related results. Section 4 is, basically, a correction to [5].

\section{Preliminaries}

Definition 2.1 (George and Veeramani [1]). A fuzzy metric space is an ordered triple $(X, M, *)$ such that $X$ is a (non-empty) set, $*$ is a continuous $t$ norm and $M$ is a fuzzy set on $X \times X \times] 0,+\infty$ [ satisfying the following conditions, for all $x, y, z \in X, s, t>0$ :

(GV1) $M(x, y, t)>0$;

(GV2) $M(x, y, t)=1$ if and only if $x=y$;

(GV3) $M(x, y, t)=M(y, x, t)$;

$(\mathrm{GV} 4) M(x, y, t) * M(y, z, s) \leq M(x, z, t+s)$;

$\left.\left.(\mathrm{GV} 5) M_{x y}:\right] 0,+\infty[\rightarrow] 0,1\right]$ is continuous, where $M_{x y}(t)=M(x, y, t)$.

The continuous $t$-norms commonly used in fuzzy logic are the minimum $(\wedge)$, the usual product $(\cdot)$, and the Lukasiewicz $t$-norm $(\mathfrak{L})$. 
If $(X, M, *)$ is a fuzzy metric space, we will say that $(M, *)$, or simply $M$, is a fuzzy metric on $X$. This terminology will also be extended along the paper in other concepts, as usual, without explicit mention.

George and Veeramani proved in [1] that every fuzzy metric $M$ on $X$ generates a topology $\tau_{M}$ on $X$ which has as a base the family of open sets of the form $\left\{B_{M}(x, \varepsilon, t): x \in X, 0<\varepsilon<1, t>0\right\}$, where $B_{M}(x, \varepsilon, t)=$ $\{y \in X: M(x, y, t)>1-\varepsilon\}$ for all $x \in X, \varepsilon \in] 0,1[$ and $t>0$.

Let $(X, d)$ be a metric space and let $M_{d}$ be a function on $\left.X \times X \times\right] 0, \infty[$ defined by

$$
M_{d}(x, y, t)=\frac{t}{t+d(x, y)} .
$$

Then $\left(X, M_{d}, \cdot\right)$ is a fuzzy metric space [1] and $M_{d}$ is called the standard fuzzy metric induced by $d$. The topology $\tau_{M}$ coincides with the topology $\tau(d)$ on $X$ deduced from $d$.

A fuzzy metric $M$ on $X$ is said to be stationary if $M$ does not depend on $t$, i.e. if for each $x, y \in X$, the function $M_{x y}(t)=M(x, y, t)$ is constant. In this case we write $M(x, y)$ instead of $M(x, y, t)$. Also, we say that a fuzzy metric $M$ on $X$ is strong (non-Archimedean) if it satisfies for each $x, y, z \in X$ and each $t>0$ the following inequality:

$$
M(x, z, t) \geq M(x, y, t) * M(y, z, t) .
$$

Definition 2.2 (Grabiec [3]). A sequence $\left\{x_{n}\right\}$ in a fuzzy metric space $(X, M, *)$ is called $G$-Cauchy if $\lim _{n} M\left(x_{n}, x_{n+p}, t\right)=1$ for each $t>0$ and each $p \in \mathbb{N}$.

This definition is equivalent to $\lim _{n} M\left(x_{n}, x_{n+1}, t\right)=1$ for all $t>0$ (see [10]).

Definition 2.3 (George and Veeramani [1.). A sequence $\left\{x_{n}\right\}$ in a fuzzy metric space $(X, M, *)$ is said to be $M$-Cauchy, or simply Cauchy, if for each $\varepsilon \in] 0,1\left[\right.$ and each $t>0$ there exists $n_{0} \in \mathbb{N}$ such that $M\left(x_{n}, x_{m}, t\right)>1-\varepsilon$ for all $n, m \geq n_{0}$ or, equivalently, $\lim _{n, m} M\left(x_{n}, x_{m}, t\right)=1$ for all $t>0$.

D. Mihet introduced in [11 a family of mappings denoted by $\Psi$, which we adapt to our context (see [5, Remark 2.13]). Then, $\Psi$ is the class of all mappings $\psi:] 0,1] \rightarrow] 0,1]$ such that $\psi$ is continuous, non decreasing and $\psi(s)>s$ for all $s \in] 0,1[$. Also, in [15] D. Wardowski denoted by $\mathcal{H}$ the 
family of mappings $\eta:] 0,1] \rightarrow[0, \infty[$ satisfying that $\eta$ is onto and strictly decreasing. On account of both classes of mappings aforementioned, we have the following definition.

Definition 2.4. Let $M$ be a fuzzy metric on $X$. A mapping $f: X \rightarrow X$ is called

(i) RT-contractive [12] if there exists $k \in] 0,1[$ such that:

$$
M(f(x), f(y), t) \geq 1-k+k M(x, y, t) \quad \text { for all } x, y \in X \text { and } t>0 .
$$

(ii) $G S$-contractive [8] if there exists $k \in] 0,1[$ such that:

$\frac{1}{M(f(x), f(y), t)}-1 \leq k\left(\frac{1}{M(x, y, t)}-1\right) \quad$ for all $x, y \in X$ and $t>0$.

(iii) fuzzy $\mathcal{H}$-contractive [15] with respect to $\eta \in \mathcal{H}$ if there exists $k \in] 0,1[$ such that:

$$
\eta(M(f(x), f(y), t)) \leq k \eta(M(x, y, t)) \quad \text { for all } x, y \in X \text { and } t>0 .
$$

(iv) fuzzy $\psi$-contractive [11, with respect to $\psi \in \Psi$, if:

$$
M(f(x), f(y), t) \geq \psi(M(x, y, t)) \quad \text { for all } x, y \in X \text { and } t>0 .
$$

The following definition is according to Definition 2.4.

Definition 2.5. Let $(X, M, *)$ be a fuzzy metric space. A sequence $\left\{x_{n}\right\}$ in $X$ is called

(i) $R T$-contractive [12] if there exists $k \in] 0,1[$ such that

$$
M\left(x_{n+1}, x_{n+2}, t\right) \geq 1-k+k M\left(x_{n}, x_{n+1}, t\right) \text { for all } n \in \mathbb{N} \text { and } t>0 .
$$

(ii) $G S$-contractive [8] if there exists $k \in] 0,1[$ such that:

$\frac{1}{M\left(x_{n+1}, x_{n+2}, t\right)}-1 \leq k\left(\frac{1}{M\left(x_{n}, x_{n+1}, t\right)}-1\right) \quad$ for all $n \in \mathbb{N}$ and $t>0$.

(iii) fuzzy $\mathcal{H}$-contractive [15] with respect to $\eta \in \mathcal{H}$ if there exists $k \in] 0,1[$ satisfying

$$
\eta\left(M\left(x_{n+1}, x_{n+2}, t\right)\right) \leq k \eta\left(M\left(x_{n}, x_{n+1}, t\right)\right) \text { for all } n \in \mathbb{N} \text { and } t>0 .
$$

(iv) fuzzy $\psi$-contractive [11], with respect to $\psi \in \Psi$, if

$$
M\left(x_{n+1}, x_{n+2}, t\right) \geq \psi\left(M\left(x_{n}, x_{n+1}, t\right)\right) \text { for all } n \in \mathbb{N} \text { and } t>0 .
$$




\section{Strictly fuzzy contractive sequences}

In this section $(X, M, *)$ is a fuzzy metric space. By a (fuzzy) contractive sequence we refer to any of the ones mentioned in Definition 2.5, when specification is not needed. The following proposition shows the relationship among the different notions of contractive sequence.

Proposition 3.1. The following chain of implications related to sequences, is satisfied:

$$
\text { RT-contractive } \Longrightarrow \text { GS-contractive } \Longrightarrow \mathcal{H} \text {-contractive } \Longrightarrow \psi \text {-contractive }
$$

Proof. The first implication is obtained with a simple computation (with the same constant $k$ ). The second one is a consequence of [15, Example 3.1]. The last one is a consequence of [4, Proposition 6].

We introduce now the following concept as a result of the discussion made in Introduction.

Definition 3.2. We will say that a sequence $\left\{x_{n}\right\}$ is strictly fuzzy $\psi$-contractive if $M\left(x_{m+1}, x_{n+1}, t\right) \geq \psi\left(M\left(x_{m}, x_{n}, t\right)\right)$ for all $n, m \in \mathbb{N}$ and $t>0$. In an analogous way are defined the concepts of strictly fuzzy contractivity for the other three concepts of contractive sequence.

Proposition 3.3. A sequence $\left\{x_{n}\right\}$ is strictly fuzzy $\psi$-contractive if and only if for each $p \in \mathbb{N}, M\left(x_{m+p}, x_{n+p}, t\right) \geq \psi\left(M\left(x_{m}, x_{n}, t\right)\right)$ holds for all $n, m \in \mathbb{N}$.

Proof. Suppose that the sequence $\left\{x_{n}\right\}$ is strictly fuzzy $\psi$-contractive and let $p \in \mathbb{N}$. Take $t>0$. With an induction process on $p$, we have for all $m, n \in \mathbb{N}$ that

$$
\begin{aligned}
M\left(x_{m+p}, x_{n+p}, t\right) & \geq \psi\left(M\left(x_{m+p-1}, x_{n+p-1}, t\right)\right) \geq \ldots \geq \psi\left(\psi^{p-1)}\left(M\left(x_{m}, x_{n}, t\right)\right)\right) \\
& \geq \psi\left(M\left(x_{m}, x_{n}, t\right)\right) .
\end{aligned}
$$

The converse is obvious.

Remark 3.4. Analogous statements can be given for the rest of the notions of strictly fuzzy contractivity.

The following proposition ensures the existence of strictly fuzzy contractive sequences. 
Proposition 3.5. Let $f: X \rightarrow X$ be a contractive mapping in any of the senses in Definition 2.4. Then, for each $x_{0} \in X$, the sequence of iterates $\left\{f^{(n)}(x)\right\}$ is strictly fuzzy contractive for the same sense.

Proof. Let $x_{0} \in X$ and suppose that $f$ is $\psi$-contractive. Put $x_{n}=f^{(n)}(x)$, $n=1,2, \ldots$ For $t>0$ we have that

$$
M\left(x_{m+1}, x_{n+1}, t\right)=M\left(f\left(x_{m}\right), f\left(x_{n}\right), t\right) \geq \psi\left(M\left(x_{m}, x_{n}, t\right)\right)
$$

for all $m, n \in \mathbb{N}$.

The proof for the other contractive concepts is analogous.

At the light of Proposition 3.1 the reader can easily obtain the same chain of implications with strictly fuzzy contractive sequences instead of the contractive ones.

The concept of strictly contractive sequence can also be given in metric spaces, as follows.

Definition 3.6. Let $(X, d)$ be a metric space. A sequence $\left\{x_{n}\right\}$ in $(X, d)$ is strictly contractive if there exists $k \in] 0,1[$ such that

$$
d\left(x_{m+1}, x_{n+1}\right) \leq k d\left(x_{m}, x_{n}\right) \text {, for all } m, n \in \mathbb{N} \text {. }
$$

It is also said that $\left\{x_{n}\right\}$ is strictly $d$-contractive. This terminology is commonly used in topology and we will use it in other concepts without explicit mention. Obviously, if $f$ is a contractive self-mapping of $(X, d)$ then $\left\{f^{n}(x)\right\}_{n}$ is strictly $d$-contractive, for each $x \in X$.

Proposition 3.7. Let $\left(\mathbb{R}, M_{d}, \cdot\right)$ be the standard fuzzy metric where $d$ is the usual Euclidean metric on $\mathbb{R}$. Then, every monotone (non decreasing or non increasing) GS-contractive sequence in $\mathbb{R}$ is strictly GS-contractive.

Proof. Suppose that $\left\{x_{n}\right\}$ is a non-decreasing $G S$-contractive sequence in $\mathbb{R}$. On account of [8, Proposition 3.9], $\left\{x_{n}\right\}$ is a $d$-contractive sequence for the same constant of contractivity, say $k \in] 0,1\left[\right.$. We will prove that $\left\{x_{n}\right\}$ is strictly $d$-contractive. Indeed, for $m, n \in \mathbb{N}$, with $m>n$, we have that

$$
d\left(x_{m+1}, x_{n+1}\right) \leq d\left(x_{m+1}, x_{m}\right)+d\left(x_{m}, x_{m-1}\right)+\ldots+d\left(x_{n+2}, x_{n+1}\right) .
$$


Since $\left\{x_{n}\right\}$ is $d$-contractive and monotone we have that

$$
\begin{aligned}
d\left(x_{m+1}, x_{n+1}\right) & \leq k d\left(x_{m}, x_{m-1}\right)+k d\left(x_{m-1}, x_{m-2}\right)+\ldots+k d\left(x_{n+1}, x_{n}\right) \\
& =k\left(d\left(x_{m}, x_{m-1}\right)+\ldots+d\left(x_{n+1}, x_{n}\right)\right) \\
& =k\left(d\left(x_{m}, x_{n}\right)\right)
\end{aligned}
$$

and so $\left\{x_{n}\right\}$ is strictly $d$-contractive. Therefore,

$$
\begin{aligned}
\frac{1}{M_{d}\left(x_{m+1}, x_{n+1}, t\right)}-1 & =\frac{t+d\left(x_{m+1}, x_{n+1}\right)}{t}-1=\frac{d\left(x_{m+1}, x_{n+1}\right)}{t} \\
& \leq \frac{k d\left(x_{m}, x_{n}\right)}{t}=k\left(\frac{1}{M_{d}\left(x_{m}, x_{n}, t\right)}-1\right),
\end{aligned}
$$

and hence $\left\{x_{n}\right\}$ is strictly $G S$-contractive.

In Example 3.9 we will construct a contractive sequence in $\mathbb{R}$, provided with its usual metric, which is not strictly contractive. Then, based on this sequence, we will give an example of an $R T$-contractive sequence (the strongest concept of contractivity, here considered) which is not strictly $R T$ contractive. Before, we need the following proposition.

Proposition 3.8. Let $(X, d)$ be a metric space such that $d(x, y)<1$ for all $x, y \in X$. Denote by $(X, N, \mathfrak{L})$ the stationary fuzzy metric space where $N(x, y)=1-d(x, y)$. Then

(i) $\tau_{N}=\tau(d)$.

(ii) $\left\{x_{n}\right\}$ is (strictly) RT-contractive in $(X, N, \mathfrak{L})$ if and only if $\left\{x_{n}\right\}$ is (strictly) d-contractive.

(iii) $\left\{x_{n}\right\}$ is $N$-Cauchy if and only if $\left\{x_{n}\right\}$ is d-Cauchy.

(iv) $(X, N, \mathfrak{L})$ is complete if and only if $(X, d)$ is complete.

Proof. It is straightforward.

It is left to the reader to introduce a similar proposition for $\left(X, M_{d}, \cdot\right)$. 
Example 3.9. (a) (A non-strictly contractive sequence). Consider the metric space $(X, d)$, where $X=\mathbb{R}$ and $d$ is the Euclidean metric. Let $a, b \in \mathbb{R}$ with $0 \leq a<b$. We define the sequence $\left\{x_{n}\right\}$ in $\mathbb{R}$, by recurrence as follows.

$$
\begin{gathered}
x_{1}=\frac{a+b}{2}, x_{2}=\frac{a+x_{1}}{2}, \\
x_{2 k-1}=\frac{x_{2 k-2}+x_{2 k-3}}{2}, \quad x_{2 k}=\frac{x_{2 k-1}+x_{2 k-3}}{2}, \text { for } k \geq 2 .
\end{gathered}
$$

The sequence $\left\{x_{n}\right\}$ is $d$-contractive. Indeed,

$d\left(x_{k+1}, x_{k}\right)=\left|x_{k+1}-x_{k}\right|=\left|x_{k+1}+x_{k-1}-x_{k-1}-x_{k}\right|=2\left|x_{k+2}-x_{k+1}\right|$,

that is, $d\left(x_{k+2}, x_{k+1}\right) \leq \frac{1}{2} d\left(x_{k+1}, x_{k}\right)$, and so $\left\{x_{n}\right\}$ is $d$-contractive. This sequence is not strictly $d$-contractive. Indeed, for $k \geq 1$, we have that

$$
\begin{aligned}
d\left(x_{4 k+2}, x_{4 k}\right) & =\left|\frac{x_{4 k+1}}{2}+\frac{x_{4 k-1}}{2}-x_{4 k}\right|=\left|\frac{x_{4 k}+x_{4 k-1}}{4}+\frac{x_{4 k-1}}{2}+x_{4 k}\right| \\
& =\frac{3}{4}\left|x_{4 k-1}-x_{4 k}\right|>\frac{1}{2}\left|x_{4 k-1}-x_{4 k}\right|=\left|x_{4 k-1}-\frac{x_{4 k}-x_{4 k-1}}{2}\right| \\
& =\left|x_{4 k-1}-x_{4 k+1}\right|=d\left(x_{4 k+1}, x_{4 k-1}\right),
\end{aligned}
$$

and hence, the sequence $\left\{x_{n}\right\}$ is not strictly contractive.

On the other hand, the sequence $\left\{x_{n}\right\}$ is convergent in $\mathbb{R}$, since every contractive sequence in a metric space is Cauchy, and $\mathbb{R}$ is complete.

(b) (A non-strictly $R T$-contractive sequence). Now, take $a=0, b=1 / 2$ in the part $(a)$ of this example. The corresponding sequence $\left\{x_{n}\right\}$, named now $\left\{y_{n}\right\}$, is a non-strictly contractive sequence in the complete metric space $([0,1 / 2], d)$ and by $(i i)$ of Proposition $3.8,\left\{y_{n}\right\}$ is a non-strictly $R T$-contractive sequence in $([0,1 / 2], N, \mathfrak{L})$. Moreover, by $(i i i)-(i v)$ of Proposition 3.8 the sequence $\left\{y_{n}\right\}$ is $N$-Cauchy and then it converges in $[0,1 / 2]$. 
Remark 3.10. After a tedious computation it can be proved that the sequence $\left\{x_{n}\right\}$ in (a) of Example 3.9 converges to $(3 a+2 b) / 5$. Indeed, by induction on $k$, it is easy to prove that

$$
x_{4 k}-x_{4 k-1}=\frac{(b-a)}{16^{k}} .
$$

Furthermore, for every $k \in \mathbb{N}$, we have that

$$
\begin{aligned}
x_{4 k} & =\frac{x_{4 k-1}+x_{4 k-3}}{2}=\frac{\left(x_{4 k-2}+x_{4 k-3}\right)+\left(x_{4 k-4}+x_{4 k-5}\right)}{4} \\
& =\frac{x_{4 k-3}+x_{4 k-5}+x_{4 k-4}+x_{4 k-5}+2 x_{4 k-4}+2 x_{4 k-5}}{8} \\
& =\frac{x_{4 k-4}+x_{4 k-5}+2 x_{4 k-5}+2 x_{4 k-4}+2 x_{4 k-5}+4 x_{4 k-4}+4 x_{4 k-5}}{16} \\
& =\frac{7 x_{4 k-4}+9 x_{4 k-5}}{16}=\frac{7 x_{4(k-1)}+9 x_{4(k-1)-1}}{16} .
\end{aligned}
$$

Now, using Equation (1), we have that

$$
x_{4 k}=\frac{7 x_{4(k-1)}+9\left(x_{4(k-1)}-\frac{(b-a)}{16^{k-1}}\right)}{16}=x_{4(k-1)}-\frac{9(b-a)}{16} \frac{1}{16^{k-1}} .
$$

Using the notation $y_{k}=x_{4 k}$, we get a recurrence sequence given by

$$
y_{k}=y_{k-1}-\frac{9(b-a)}{16^{k}}, \quad \text { for } k=2,3 \ldots
$$

Now, one can prove that $y_{1}=x_{4}=\frac{9 a+7 b}{16}$, and then, by a few calculations one obtains

$$
y_{k}=\frac{3 a+2 b}{5}-\frac{3(a-b)}{5} \frac{1}{16^{k}} .
$$

Therefore the sequence $\left\{y_{n}\right\}$, obviously, converges to $(3 a+2 b) / 5$. Our conclusion holds by the fact that all subsequences of a convergent sequence converge to the same point.

Now we are interested in the relationship between fuzzy contractive sequences and Cauchy sequences. The most general result that we can give is the following proposition, which states that every fuzzy contractive sequence of Definition 2.5 is $G$-Cauchy. 
Proposition 3.11. Every $\psi$-contractive sequence is G-Cauchy.

Proof. Let $\left\{x_{n}\right\}$ be a $\psi$-contractive sequence. Then, for $t>0$ we have that

$$
M\left(x_{n}, x_{n+1}, t\right) \geq \psi\left(M\left(x_{n-1}, x_{n}, t\right)\right) \geq \ldots \geq \psi^{n)}\left(M\left(x_{1}, x_{2}, t\right)\right),
$$

for all $t>0$. Now for each $s \in] 0,1\left[\right.$ it is easy to verify that $\lim _{n} \psi^{n)}(s)=1$, and then $\lim _{n} M\left(x_{n}, x_{n+1}, t\right)=1$ for each $t>0$.

In the following example we show a $\psi$-contractive sequence which is not Cauchy.

Example 3.12 (A $\psi$-contractive sequence which is not Cauchy). Consider the real line $\mathbb{R}$ endowed with the usual Euclidean metric $d$. We define the mapping $\tilde{\psi}:[0,1] \rightarrow[0,1]$ as follows.

Consider the partition $\{[\ell /(\ell+1),(\ell+1) /(\ell+2)[: \ell=0,1,2, \ldots\}$ of $[0,1[$. We define

$$
\tilde{\psi}(s)=\left\{\begin{array}{cc}
\frac{\ell+1}{\ell+3} \cdot s+\frac{2 \ell+3}{(\ell+2)(\ell+3)}, & \text { for } s \in\left[\frac{\ell}{\ell+1}, \frac{\ell+1}{\ell+2}[,\right. \\
1 & \text { for } s=1 .
\end{array}\right.
$$

Let $\psi$ the restriction of $\tilde{\psi}$ to $] 0,1]$. Then, it is easy to verify that $\psi(s)>s$ for all $s \in] 0,1[$, and that $\psi$ is non-decreasing on $[0,1]$. Also $\psi$ is continuous on $] 0,1\left[\right.$. We will see that $\psi$ is continuous at $s=1$. Suppose that $\left\{s_{j}\right\}$ is a sequence in $[0,1]$ that converges to 1 . We will see that $\left\{\psi\left(s_{j}\right)\right\}$ converges to 1 .

Let $0<\varepsilon<1$. Choose $\ell_{0} \in \mathbb{N}$ such that $\ell_{0} /\left(\ell_{0}+3\right)>1-\varepsilon$. Then

$\psi\left(\frac{\ell_{0}}{\ell_{0}+1}\right)=\frac{\ell_{0}+1}{\ell_{0}+3} \cdot \frac{\ell_{0}}{\ell_{0}+1}+\frac{2 \ell_{0}+2}{(\ell+2)(\ell+3)}>\frac{\ell_{0}+1}{\ell_{0}+3} \cdot \frac{\ell_{0}}{\ell_{0}+1}=\frac{\ell_{0}}{\ell_{0}+3}>1-\varepsilon$

Take $\delta>0$ such that $\ell_{0} /\left(\ell_{0}+1\right)<\delta<1$. Then, there exists $j_{0} \in \mathbb{N}$ such that $\left.\left.s_{j} \in\right] \delta, 1\right]$ for $j \geq j_{0}$, since $\left\{s_{j}\right\}$ converges to 1 . Hence, for $j \geq j_{0}$ we have that $\psi\left(s_{j}\right) \geq \psi(\delta) \geq \psi\left(\frac{\ell_{0}}{\ell_{0}+1}\right)>1-\varepsilon$. Hence, $\psi$ is continuous at $s=1$.

Consider the sequence (harmonic series) $\left\{x_{n}\right\}$ where $x_{n}=\sum_{i=1}^{n} 1 / i$. It is well known that $\left\{x_{n}\right\}$ is a $G$-Cauchy sequence which is not Cauchy in the standard fuzzy metric space $\left(\mathbb{R}, M_{d}, \cdot\right)$ (see [1]). Then it is clear that $\left\{x_{n}\right\}$ is 
$G$-Cauchy in the stationary fuzzy metric space $\left(X, M_{1}, \cdot\right)$ where $M_{1}(x, y)=$ $\frac{1}{1+d(x, y)}$ and it is almost immediate that $\left\{x_{n}\right\}$ is not Cauchy in $\left(\mathbb{R}, M_{1}, \cdot\right)$. Now we will prove that $\left\{x_{n}\right\}$ is $\psi$-contractive.

For $n \in \mathbb{N}$ we have that

$$
d\left(x_{n}, x_{n+1}\right)=\frac{1}{n+1}, \quad d\left(x_{n+1}, x_{n+2}\right)=\frac{1}{n+2} .
$$

Then,

$$
M_{1}\left(x_{n}, x_{n+1}\right)=\frac{1}{1+\frac{1}{n+1}}=\frac{n+1}{n+2}, \quad M_{1}\left(x_{n+1}, x_{n+2}\right)=\frac{1}{1+\frac{1}{n+2}}=\frac{n+2}{n+3} .
$$

Now, since

$$
\begin{aligned}
\psi\left(M_{1}\left(x_{n}, x_{n+1}\right)\right) & =\psi\left(\frac{n+1}{n+2}\right)=\frac{n+2}{n+4} \cdot \frac{n+1}{n+2}+\frac{2(n+1)+3}{(n+3)(n+4)}= \\
& =\frac{n+1}{n+2} \in\left[\frac{n+1}{n+2}, \frac{n+2}{n+3}[\right.
\end{aligned}
$$

we have that

$$
M_{1}\left(x_{n+1}, x_{n+2}\right)=\frac{n+2}{n+3} \geq \psi\left(M_{1}\left(x_{n}, x_{n+1}\right)\right),
$$

and so $\left\{x_{n}\right\}$ is $\psi$-contractive.

Remark 3.13. The sequence $\left\{x_{n}\right\}$ of Example 3.12 is not strictly fuzzy $\psi$ contractive in $\left(\mathbb{R}, M_{1}, \cdot\right)$. Indeed, $d\left(x_{2}, x_{4}\right)=7 / 12$ and $d\left(x_{3}, x_{5}\right)=9 / 20$. Then $M_{1}\left(x_{3}, x_{5}\right)=20 / 29$ and $M_{1}\left(x_{2}, x_{4}\right)=12 / 19$. Since $12 / 19 \in[1 / 2,2 / 3[$, then

$$
\psi\left(M_{1}\left(x_{2}, x_{4}\right)\right)=\psi(12 / 19)=\frac{2}{4} \cdot \frac{12}{19}+\frac{7}{20}=\frac{373}{380} .
$$

Then, $M_{1}\left(x_{3}, x_{5}\right)=20 / 29<373 / 380=\psi\left(M_{1}\left(x_{2}, x_{4}\right)\right)$, and so, $\left\{x_{n}\right\}$ is not strictly fuzzy $\psi$-contractive. Furthermore, it can be proved that for each $n \in \mathbb{N}$ there exists $m \in \mathbb{N}$ with $m>n$ satisfying $M_{1}\left(x_{n+1}, x_{m+1}\right)<$ $\psi\left(M_{1}\left(x_{n}, x_{m}\right)\right)$. Indeed, fix $n \in \mathbb{N}$. Since $\left\{x_{n}\right\}$ is a sequence of positive terms 
which diverges to infinity, then we can find $m>n$ satisfying $\sum_{i=n+2}^{m+1} 1 / i \geq 1$. Then,

$$
d\left(x_{n+1}, x_{m+1}\right)=\sum_{i=n+2}^{m+1} \frac{1}{i} \geq 1, \quad d\left(x_{n}, x_{m}\right)=\sum_{i=n+1}^{m} \frac{1}{i}>\sum_{i=n+2}^{m+1} \frac{1}{i} \geq 1 .
$$

Thus,

$$
M_{1}\left(x_{n+1}, x_{m+1}\right)=\frac{1}{1+d\left(x_{n+1}, x_{m+1}\right)} \leq \frac{1}{2}
$$

and

$$
M_{1}\left(x_{n}, x_{m}\right)=\frac{1}{1+d\left(x_{n}, x_{m}\right)}<\frac{1}{2} .
$$

As $M_{1}\left(x_{n}, x_{m}\right) \in\left[0,1 / 2\left[\right.\right.$, we take $\ell=0$ to compute $\psi\left(M_{1}\left(x_{n}, x_{m}\right)\right)$, and so, since $M_{1}\left(x_{n}, x_{m}\right)>0$, we have that

$$
\psi\left(M_{1}\left(x_{n}, x_{m}\right)\right)=\frac{1}{3} M_{1}\left(x_{n}, x_{m}\right)+\frac{1}{2}>\frac{1}{2} \geq M\left(x_{n+1}, x_{m+1}\right) .
$$

Remark 3.14. Recently in [14 the following contractive condition, related with the above ones, has been given.

Denote by $\mathcal{Z}$ the family of all functions $\zeta:[0,1] \times] 0,1] \rightarrow \mathbb{R}$ satisfying $\zeta(t, s)>s$ for all $t, s \in] 0,1[$. Let $(X, M, *)$ be a fuzzy metric space and let $f: X \rightarrow X$ be a mapping. Then, $f$ is called a fuzzy $\mathcal{Z}$-contractive mapping with respect to $\zeta$ if $M(f(x), f(y), t) \geq \zeta(M(f(x), f(y), t), M(x, y, t))$ for all $x, y \in X, f(x) \neq f(y), t>0$ where $\zeta \in \mathcal{Z}$. According to this definition, we can say that a sequence $\left\{x_{n}\right\}$ in $X$ is $\mathcal{Z}$-contractive with respect to $\zeta \in \mathcal{Z}$ if $M\left(x_{n+1}, x_{n+2}, t\right) \geq \zeta\left(M\left(x_{n+1}, x_{n+2}, t\right), M\left(x_{n}, x_{n+1}, t\right)\right)$ for all $n \in \mathbb{N}$ and $t>0$.

In [14] it is observed that every $\psi$-contractive mapping is fuzzy $\mathcal{Z}$-contractive wih respect to the function $\zeta_{M}$ given by $\zeta_{M}(t, s)=\psi(s)$ for all $\left.\left.s, t \in\right] 0,1\right]$. Then it is immediate that every $\psi$-contractive sequence is $\mathcal{Z}$-contrative sequence with respect to $\zeta_{M}$. Therefore the sequence $\left\{x_{n}\right\}$ constructed in Example 3.12 is a fuzzy $\mathcal{Z}$-contractive sequence which is not Cauchy.

\section{Correction to Lemma 3.2 of 5}

Lemma 3.2 of [5] asserts that if $\left\{x_{n}\right\}$ is a $\psi$-contractive sequence satisfying $\bigwedge_{t>0} M\left(x_{1}, x_{2}, t\right)>0$ then $\left\{x_{n}\right\}$ is Cauchy. Clearly, at the light of Example 
3.12 this assertion is false. Nevertheless one can observe on the proof of this lemma that this assertion is true if we assume that $\left\{x_{n}\right\}$ is strictly fuzzy $\psi$-contractive.

Then, accordingly to the last paragraph, Corollary 3.8 and Lemma 3.12 of [5] must also be corrected as show the next propositions.

Proposition 4.1. (Correction of [5, Corollary 3.8]). Let $(X, M, *)$ be a fuzzy metric space such that $\bigwedge_{t>0} M(x, y, t)>0$ for all $x, y \in X$. Then every strictly fuzzy $\psi$-contractive sequence is a Cauchy sequence.

Proposition 4.2. (Correction of [5, Lemma 3.12]). Let $(X, M, *)$ be a strong fuzzy metric space. Then every strictly fuzzy $\psi$-contractive sequence is a Cauchy sequence.

Remark 4.3. The fixed point theorems of [5] remain valid, since the sequence of iterates $\left\{f^{(n)}\left(x_{0}\right)\right\}$ for each $x_{0} \in X$, for a fuzzy $\psi$-contractive mapping $f$ of $X$, is a strictly fuzzy $\psi$-contractive sequence by Proposition 3.5 .

At the light of Proposition 4.2, we propose to investigate the next problem.

Problem 4.4. Is every strictly fuzzy $\psi$-contractive sequence a Cauchy sequence?

\section{Acknowledgements}

The authors are very grateful to the reviewers for their valuable suggestions which have helped to improve the content of the paper.

Valentín Gregori acknowledges the support of Ministry of Economy and Competitiveness of Spain under Grant MTM 2015-64373-P(MINECO/FEDER,UE).

Juan José Miñana acknowledges the support of Programa Operatiu FEDER 2014-2020 de les Illes Balears (50\%), by project ref. PROCOE/4/2017 (Direccio General d Innovacio i Recerca, Govern de les Illes Balears), and of project ROBINS. The latter has received research funding from the EU H2020 framework under GA 779776. This publication reflects only the authors views and the European Union is not liable for any use that may be made of the information contained therein.

[1] A. George and P. Veeramani, On some results in fuzzy metric spaces, Fuzzy Sets and Systems 64 (1994), 395-399. 
[2] A. George and P. Veeramani, Some theorems in fuzzy metric spaces, J. Fuzzy Math 3 (1995), 933-940.

[3] M. Grabiec, Fixed points in fuzzy metric spaces, Fuzzy Sets and Systems 27 (1989), 385-389.

[4] V. Gregori and J.-J. Miñana, Some remarks on fuzzy contractive mappings, Fuzzy Sets and Systems 251 (2014), 101-103.

[5] V. Gregori and J.-J. Miñana, On fuzzy $\psi$-contractive sequences and fixed point theorems, Fuzzy Sets and Systems 300 (2016), 93-101.

[6] V. Gregori, J.-J. Miñana, S. Morillas, and A. Sapena, Cauchyness and convergence in fuzzy metric spaces, RACSAM 111 (2017), 25-37.

[7] V. Gregori and S. Romaguera, Some properties of fuzzy metric spaces, Fuzzy Sets and Systems 115 (2000), 485-489.

[8] V. Gregori and A. Sapena, On fixed-point theorems in fuzzy metric spaces, Fuzzy Sets and Systems 125 (2002), 245-252.

[9] K. Kramosil and J. Michalek, Fuzzy metric and statistical metric spaces, Kybernetika 11 (1975), 336-344.

[10] D. Mihet, On fuzzy contractive mappings in fuzzy metric spaces, Fuzzy Sets and Systems 158 (2007), 915-921.

[11] D. Mihet, Fuzzy $\psi$-contractive mappings in non-archimedean fuzzy metric spaces, Fuzzy Sets and Systems 159 (2008), 739-744.

[12] S. Romaguera and P. Tirado, Contraction maps on Ifgm-spaces with application to Recurrence Equations of Quicksort, Electronic Notes in Theoretical Computer Science 225 (2009), 339-342.

[13] H. Sherwood, On the completion of probabilistic metric spaces, Z. Wahrschein-lichkeitstheoric verw. Geb 6 (1966), 62-64.

[14] S. Shukla, D. Gopal, and W. Sintunavarat, A new class of fuzzy contractive mappings and fixed point theorems, Fuzzy Sets and Systems 350 (2018), 85-94.

[15] D. Wardowski, Fuzzy contractive mappings and fixed points in fuzzy metric spaces, Fuzzy Sets and Systems 222 (2013), 108-114. 